PROCEEDINGS OF THE

AMERICAN MATHEMATICAL SOCIETY

Volume 129, Number 2, Pages 559-566

S 0002-9939(00)05575-

Article electronically published on October 12, 2000

\title{
TWIST DECOMPOSITIONS OF GLUING HOMEOMORPHISMS OF PLANAR HEEGAARD DIAGRAMS OF GENUS TWO
}

\author{
YOSHIKO SAKATA, MITSUYUKI OCHIAI, AND KAYO SATOH \\ (Communicated by Ronald Fintushel)
}

\begin{abstract}
We will give a very simple algorithm to decompose a gluing homeomorphism of a planar Heegaard diagram of genus two into Dehn twists associated with the canonical base.
\end{abstract}

\section{INTRODUCTION}

Let $H$ be a handlebody of genus $g$ standardly embedded in the standard Euclidean 3-space $R^{3}$. Let $v=\left\{v_{1}, v_{2}, \ldots, v_{g}\right\}$ be a standard complete system of meridians of $\partial H=F_{g}$ and let $x=\left\{x_{1}, x_{2}, \ldots, x_{g}\right\}$ be a collection of mutually disjoint circles on $F_{g}$ such that each circle $x_{i}$ intersects $v_{j}$ transversely at only one point if $i=j$ and it is disjoint from $v_{j}$ if $i \neq j$. Moreover assume that each $x_{i}$ bounds a disk in the complement of $H$ in $R^{3}$. The pair $\{v, x\}$ is called a standard meridianlongitude system of $H$. Let $L=\left\{K_{1}, K_{2}, \ldots, K_{g}\right\}$ be a collection of mutually disjoint circles on $F_{g}$. Then $L$ is called a $g$-bridge link if each connected component $K_{i}$ of $L$ always intersects $x_{i}$ transversely at only one point, and $K_{i} \cap x_{j}=\emptyset$ if $i \neq j$ $(i, j=1,2, \ldots, g)$.

Let $M$ be a connected orientable 3-manifold obtained by an integral Dehn surgery along a $g$-bridge link $L$ with $g$-components. Then $M$ has a Heegaard diagram $\left(F_{g} ; v, w\right)$, where $\{v, x\}$ (resp. $\{w, y\}$ ) is a standard meridian-longitude system of $H_{1}$ (resp. $H_{2}$ ). The diagram has the following properties:

(1) each meridian $w_{i}$ is identified with $K_{i}$,

(2) each longitude $x_{j}$ is identified with $y_{j}$ as a set, where $1 \leq i, j \leq g$.

Let $h$ be a self-homeomorphism of $F_{g}$ induced from a homeomorphism $\partial H_{2} \rightarrow$ $\partial H_{1}$ such that $h\left(w_{i}\right)=K_{i}(i=1,2, \ldots, g)$. This one $h$ gives a gluing homeomorphism of a Heegaard splitting of $M$. We call $\left(F_{g} ; v, L\right)$ a planar Heegaard diagram ([] $)$. In this paper, we study a method to decompose $h$ into a product of canonical Dehn twists. Generally speaking, such a method was first given by Lickorish L1 L2 L3] but, given a gluing homeomorphism, to decompose it into such a product is very difficult. We can simplify the method for a gluing homeomorphism of a planar Heegaard diagram.

Received by the editors January 5, 1999 and, in revised form, April 30, 1999. 1991 Mathematics Subject Classification. Primary 57M25; Secondary 57M35.

Key words and phrases. 2-bridge link, Dehn surgery, Dehn twist, Heegaard diagram. 


\section{DEHN SURGERIES ALONG A 2-BRIDGE LINK WITH 2-COMPONENTS}

Let $F_{2}$ be a closed connected orientable surface of genus two, and $a_{1}, b_{1}, a_{2}, b_{2}$, and $c$ be the circles illustrated in Figure 1. Moreover, let $c_{1}$ (resp. $c_{2}$ ) be the front segment (resp. back segment) of the circle $c$ as illustrated in Figure 1.

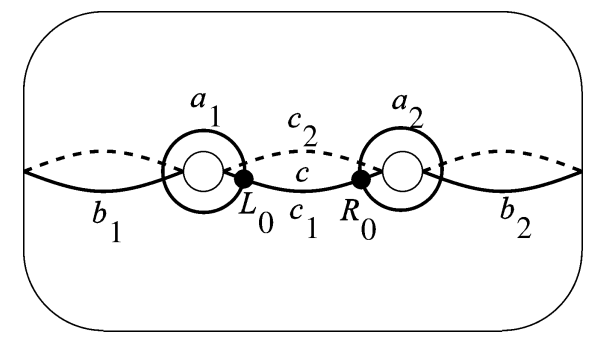

FIGURE 1.

Let $L=\left\{K_{1}, K_{2}\right\}$ be a 2-bridge link with 2-components with the canonical form $(p, q)$, where $p$ and $q$ are relatively prime integers such that $p=2 m$ and $q=$ $2 n+1(m, n \geq 0)$. Note that we also consider even a case of $p<q$ as the canonical form for the sake of convenience, because the case results with an intermediate stage under series of decomposing deformations. We denote the point $c \cap a_{1}$ (resp. $\left.c \cap a_{2}\right)$ by $L_{0}$ (resp. $\left.R_{0}\right)$. Then we can assume that $K_{1}$ (resp. $K_{2}$ ) always intersects $a_{1}$ (resp. $a_{2}$ ) transversely at only the point $L_{0}\left(\right.$ resp. $\left.R_{0}\right), K_{1} \cap a_{2}=\emptyset, K_{2} \cap a_{1}=\emptyset$, $K_{L_{0}} \cap b_{1}=\emptyset$ and $K_{R_{0}} \cap b_{2}=\emptyset$, where $K_{L_{0}}$ (resp. $K_{R_{0}}$ ) is the closure of the connected component including $L_{0}\left(\right.$ resp. $\left.R_{0}\right)$ among $K_{1}-\left(b_{1} \cup b_{2}\right)\left(\operatorname{resp} . K_{2}-\left(b_{1} \cup b_{2}\right)\right)$.

Next, let $\left\{\tilde{K}_{1}, \tilde{K}_{2}\right\}$ be a collection of surgery circles which are given by an integral Dehn surgery along $L$. That is, we can assume that $\tilde{K}_{1}=\sigma_{2}^{r}\left(K_{1}\right)$ and $\tilde{K}_{2}=\sigma_{4}^{s}\left(K_{2}\right)$ for certain integers $r$ and $s$ (see later definitions for $\sigma_{2}$ and $\sigma_{4}$ ). Then the Dehn surgery is denoted by $L\left((p, q)^{+1} ; r, s\right)$, where $\tilde{K}_{1}$ (resp. $\tilde{K}_{2}$ ) is a circle on $F_{2}$ such that it departs from $L_{0}$ (resp. $\left.R_{0}\right)$, goes around $a_{1}$ (resp. $a_{2}$ ) only $r$ (resp. $s$ ) times, goes in the upward (resp. downward) direction on the front surface of $F_{2}$, and comes back to $L_{0}$ (resp. $R_{0}$ ) from the back surface of $F_{2}$. Moreover, with the intermediate stage $\tilde{K}_{1}$ (resp. $\tilde{K}_{2}$ ) also intersects $b_{1}|m-1|$ (resp. $m$ ) times, $b_{2} m$ (resp. $|m-1|)$ times, $c_{1} n$ times, and $c_{2} n$ times. When counting the numbers of intersection, we ignore the end points $L_{0}$ (resp. $\left.R_{0}\right)$. If $r, s>0$ (resp. $r, s<0$ ), then they go around $a_{1}$ and $a_{2}$ clockwise (resp. counterclockwise).

For the sake of simplicity, later we denote $L\left((p, q)^{\epsilon} ; 0,0\right)$ simply by $L(p, q)^{\epsilon}$.

Let $\Psi$ be the straight line in $R^{3}$ which intersects $F_{2}$ at 6 points including $L_{0}$ and $R_{0}$ in Figure 2 . Then $L(p, q)^{-1}$ denotes the Dehn surgery induced by surgery circles obtained from $\tilde{K}_{1}$ and $\tilde{K}_{2}$ by rotating $F_{2}$ around $\Psi$ only $\pi$ and then by reflecting $F_{2}$.

We note that when $\epsilon= \pm 1$ and $m=n=0, L(0,1)^{\epsilon}$ is as shown in Figure 5 (5). As shown in the figure, $\tilde{K}_{1}$ (resp. $\tilde{K}_{2}$ ) does not intersect $c_{1}$ and $c_{2}$, namely it intersects $c_{1} 0$ times and $c_{2} 0$ times.

Moreover we define $L(0,1)$ as the last figure in Figure 6 . 


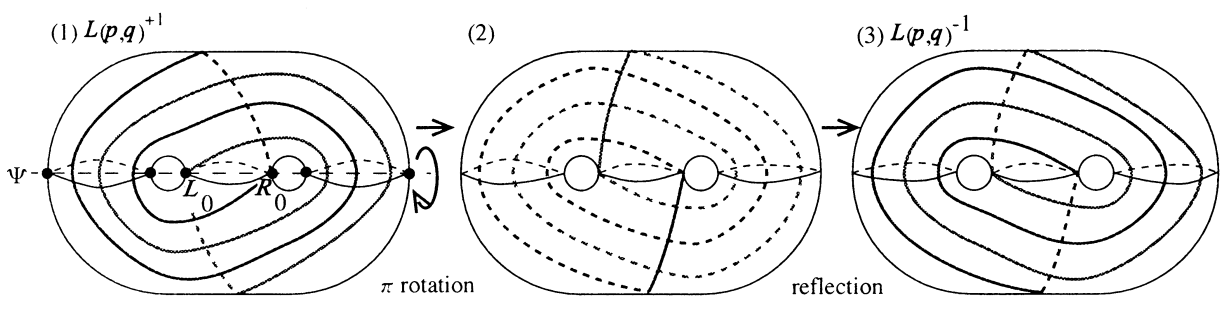

FiguRE 2. $L(p, q)^{-1}$.

\section{Decomposition of a homeomorphism into canonical Dehn twists}

Let $\sigma_{1}, \sigma_{2}, \sigma_{3}, \sigma_{4}, \sigma_{5}$ be Dehn twists along $b_{1}, a_{1}, c, a_{2}$, and $b_{2}$ respectively.

Let $d_{0}, d_{1}, d_{-1}, d_{2}$ be circles illustrated in Figure 3 , and let $D_{0}, D_{1}, D_{-1}, D_{2}$ be Dehn twists along $d_{0}, d_{1}, d_{-1}, d_{2}$ respectively. Then we have the following lemma using Lemmata in [L1, L2, L3]:

Lemma 1. $D_{0}=\sigma_{1}^{-1} \sigma_{2}^{-1} \sigma_{3}^{-1} \sigma_{5} \sigma_{4} \sigma_{5}^{-1} \sigma_{3} \sigma_{2} \sigma_{1}$,

$$
\begin{aligned}
& D_{1}=\sigma_{5} \sigma_{4} \sigma_{3}^{-1} \sigma_{2}^{-1} \sigma_{1} \sigma_{2} \sigma_{3} \sigma_{4}^{-1} \sigma_{5}^{-1}, \\
& D_{-1}=\sigma_{5}^{-1} \sigma_{4}^{-1} \sigma_{3} \sigma_{2} \sigma_{1} \sigma_{2}^{-1} \sigma_{3}^{-1} \sigma_{4} \sigma_{5}, \\
& D_{2}=\sigma_{5}^{2} \sigma_{4} \sigma_{5}^{2} \sigma_{4}^{2} \sigma_{5}^{2} \sigma_{4} \sigma_{5}^{2} .
\end{aligned}
$$
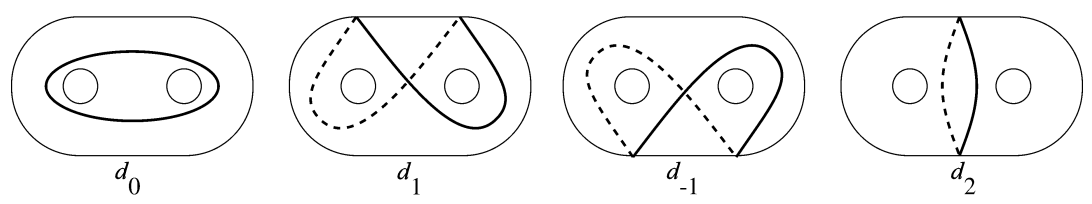

FiguRE 3.

Let $\alpha$ be any product of Dehn twists on $F_{2}$ and $L\left((p, q)^{\epsilon} ; r, s\right)$ be any integral Dehn surgery along $L$, where $\epsilon= \pm 1$. Then we can get new surgery circles $\tilde{K}_{1}^{\prime}$ and $\tilde{K}_{2}^{\prime}$ such that $\tilde{K}_{1}^{\prime}=\alpha\left(\tilde{K}_{1}\right)$ and $\tilde{K}_{2}^{\prime}=\alpha\left(\tilde{K}_{2}\right)$ and denote the resulting Dehn surgery by $L\left(\left(p^{\prime}, q^{\prime}\right)^{\epsilon} ; r^{\prime}, s^{\prime}\right)$. The notation $L\left((p, q)^{\epsilon} ; r, s\right) \rightarrow \alpha \rightarrow L\left(\left(p^{\prime}, q^{\prime}\right)^{\epsilon} ; r^{\prime}, s^{\prime}\right)$ denotes such the changing process. Then we have:

Lemma 2. $L\left((p, q)^{\epsilon} ; r, s\right) \rightarrow \sigma_{2}^{-r} \sigma_{4}^{-s} \rightarrow L(p, q)^{\epsilon}$.

From now on, for any $L(p, q)^{\epsilon}$ let $\tilde{L}=\tilde{K}_{1} \cup \tilde{K}_{2}$, and let $A=b_{1} \cap \tilde{L}, B=b_{2} \cap \tilde{L}$, $C_{1}=\left(c_{1}-\left\{L_{0}, R_{0}\right\}\right) \cap \tilde{L}$, and $C_{2}=\left(c_{2}-\left\{L_{0}, R_{0}\right\}\right) \cap \tilde{L}$. Then it is easily seen that $|A|=p-1,|B|=p-1$, and $\left|C_{1}\right|=\left|C_{2}\right|=q-1$.

Lemma 3. If $p-2 q>0, L(p, q)^{ \pm 1} \rightarrow D_{0}^{\mp 1} \rightarrow L(p-2 q, q)^{ \pm 1}$.

Proof. (See Figure 4.) Let $D=\tilde{L} \cap d_{0}^{+} \cup L_{0}$, and $E=\tilde{L} \cap d_{0}^{-} \cup R_{0}$, where $d_{0}=d_{0}^{+} \cup d_{0}^{-}$ and $d_{0}^{+}$(resp. $d_{0}^{-}$) is the upper (resp. lower) half of $d_{0}$ in (1). Then we have that $|D|=q$ and $|E|=q$. Next let $F=\tilde{L}^{\prime} \cap b_{1}$ and $G=\tilde{L}^{\prime} \cap b_{2}$ in (5). We can see $|F|=|A|-|D|-|E|,|G|=|B|-|D|-|E|$ and get $|F|=|G|=(p-2 q)-1$ in (2). $|F|$ and $|G|$ are held from (2) to (5). Then $|F|=|G|=(p-2 q)-1(\geq 1)$ in (5) because $p-2 q>0$ and $p$ is even. Furthermore we can easily verify that $\left|C_{1}\right|$ and $\left|C_{2}\right|$ are invariant. Hence we can get that $p^{\prime}=p-2 q$ and $q^{\prime}=q$. 

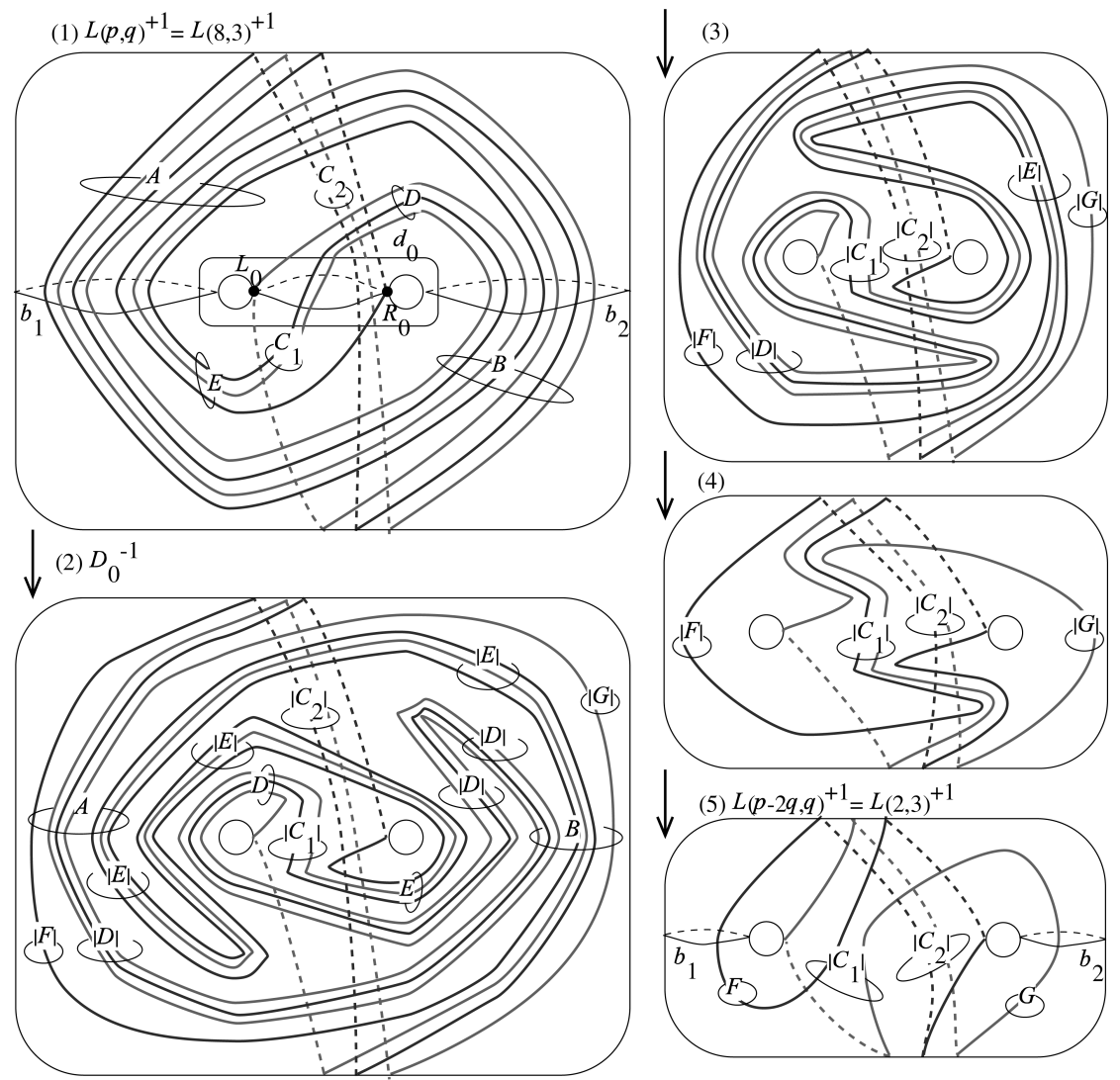

FIgURE 4.

Lemma 4. If $\frac{p}{2}<q<p-1, L(p, q)^{ \pm 1} \rightarrow \sigma_{2}^{ \pm 2} \sigma_{4}^{ \pm 2} D_{0}^{\mp 1} \rightarrow L(2 q-p, q)^{\mp 1}$.

Lemma 5. If $q=p-1$ and $p-2>0, L(p, q)^{ \pm 1} \rightarrow D_{\mp 1}^{ \pm 1} \rightarrow L(p-2, q-2)^{ \pm 1}$.

Lemma 6. If $q=p+1$ and $p-2 \geq 0, L(p, q)^{ \pm 1} \rightarrow D_{\mp 1}^{\mp 1} \rightarrow L(p-2, q-2)^{ \pm 1}$.

Proof. General cases are abbreviated. Especially in $p=2$, we get $L(0,1)^{ \pm 1}$ from $L(2,3)^{ \pm 1}$ in Figure 5.

Lemma 7. If $p+1<q<2 p, L(p, q)^{ \pm 1} \rightarrow D_{2}^{ \pm 1} \rightarrow L(p, 2 p-q)^{\mp 1}$.

Lemma 8. If $2 p<q, L(p, q)^{ \pm 1} \rightarrow D_{2}^{ \pm 1} \rightarrow L(p, q-2 p)^{ \pm 1}$.

Lemma 9. $L(2,1)^{ \pm 1} \rightarrow D_{\mp 1}^{ \pm 1} \rightarrow L(0,1)^{\mp 1}$ and $L(0,1)^{ \pm 1} \rightarrow \sigma_{2}^{ \pm 1} \sigma_{4}^{ \pm 1} \rightarrow L(0,1)$.

By this lemma, we get the last figure $L(0,1)$. Finally, we have the following:

Main Theorem. Let $M$ be a closed connected orientable 3-manifold given by an integral Dehn surgery $L\left((p, q)^{\epsilon} ; r, s\right)$, where $\epsilon= \pm 1$, along a 2-bridge link with 2components. Then there exists a simple algorithm to get $\Pi$ such that $L\left((p, q)^{\epsilon} ; r, s\right)$ $\rightarrow \Pi \rightarrow L(0,1)$, where $\Pi$ is a product of canonical Dehn twists. The algorithm is given by the following procedure: 


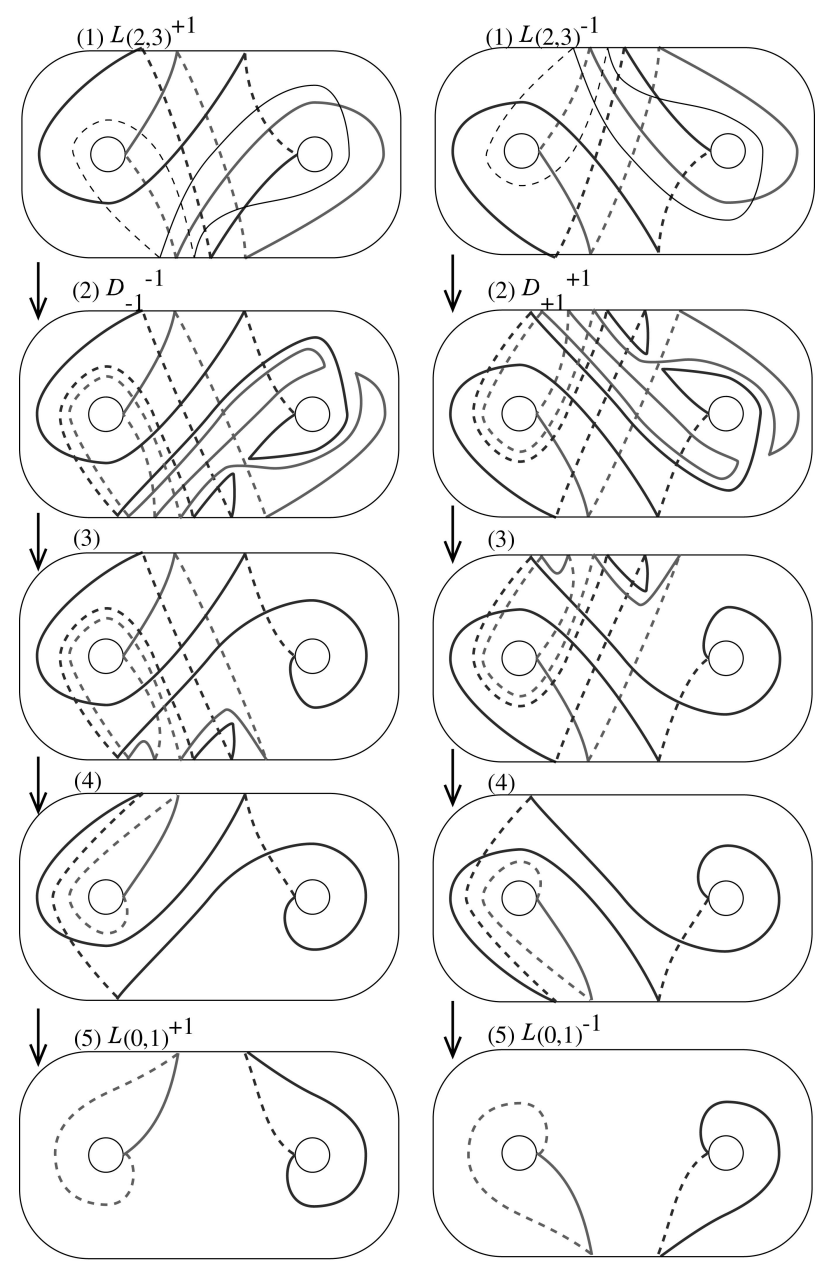

Figure 5.

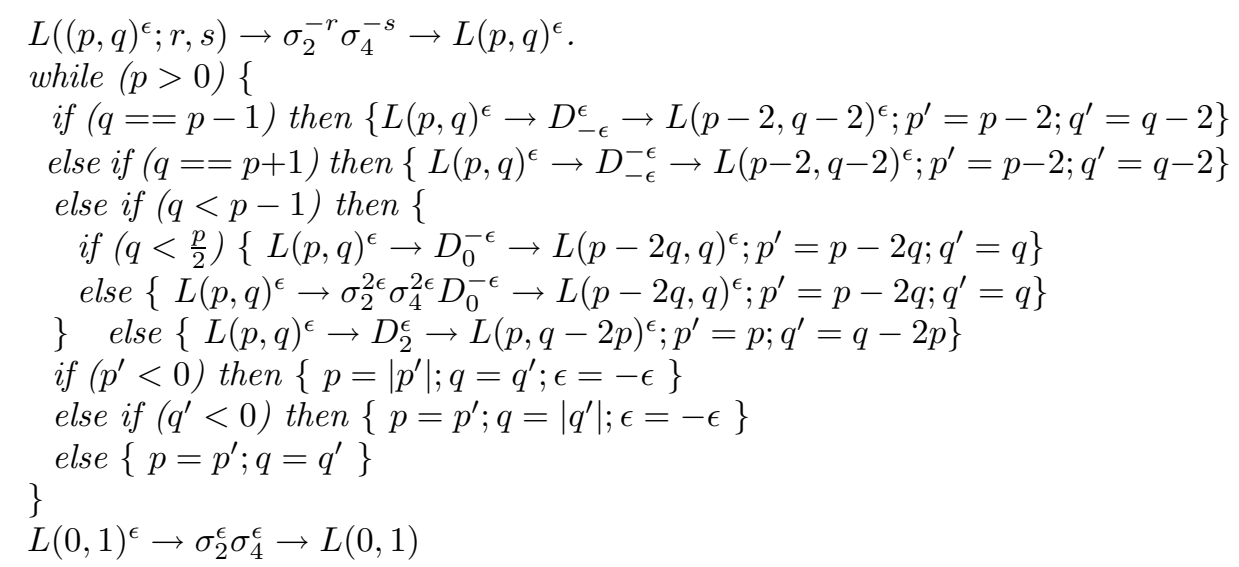




\section{EXAMPLES AND FINAL REMARKS}

Example 1. Poincaré homology 3 -sphere $\Sigma$ is given by $L(8,3)^{+1}$ and so we can decompose the gluing homeomorphism $h$ of $\Sigma$. The decomposing process is as follows:

$$
L(8,3)^{+1} \rightarrow D_{0}^{-1} \rightarrow L(2,3)^{+1} \rightarrow D_{-1}^{-1} \rightarrow L(0,1)^{+1} \rightarrow \sigma_{2} \sigma_{4} \rightarrow L(0,1) .
$$

Then we have that $L(8,3)^{+1} \rightarrow \sigma_{2} \sigma_{4} D_{-1}^{-1} D_{0}^{-1} \rightarrow L(0,1)$ (see Figure 6). Hence, we have the following decomposition of $h$ given by $h\left(w_{i}\right)=K_{i}(i=1,2)$ such that

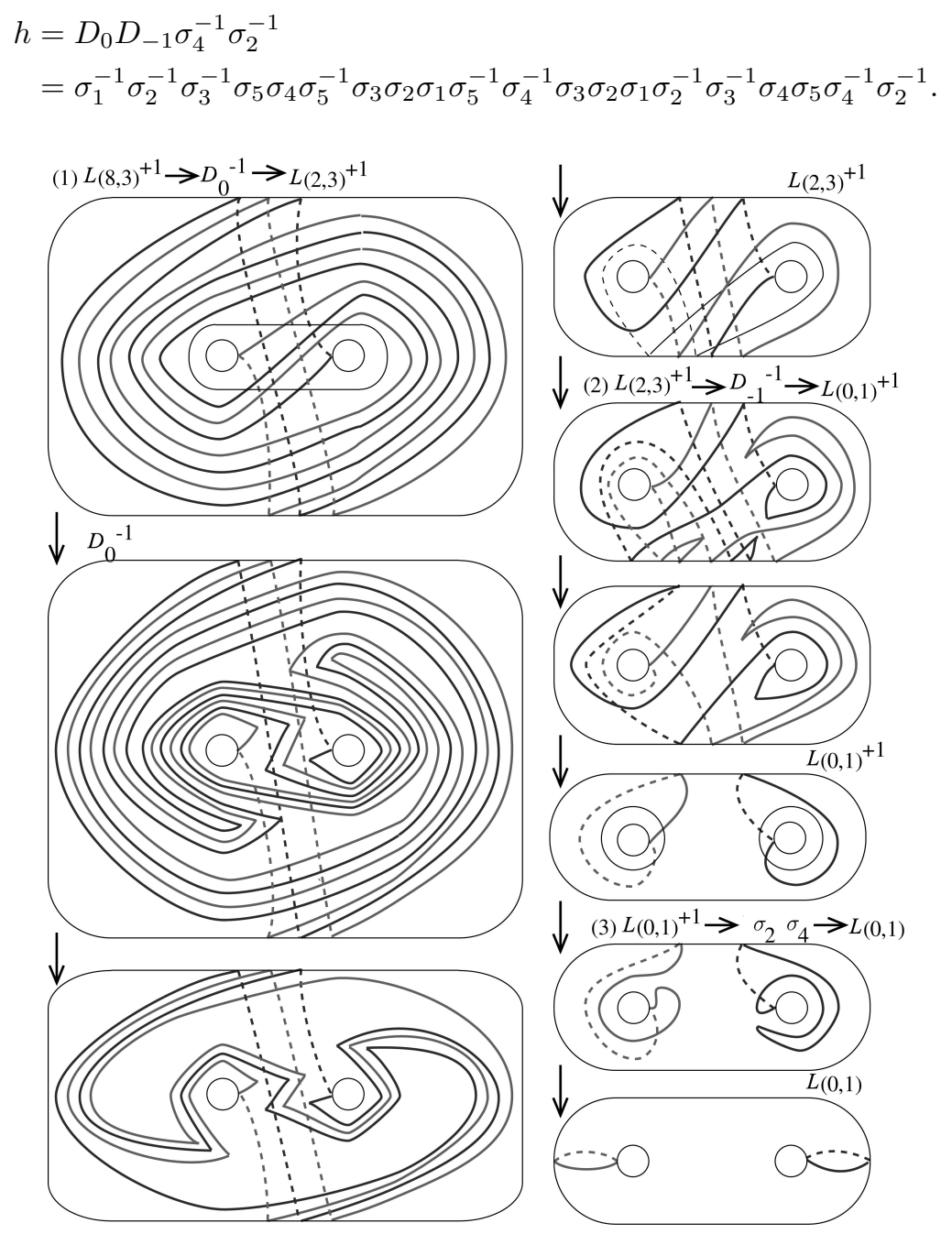

Figure 6. Poincaré homology 3-sphere.

Example 2. We give another example with the same $p, L(8,11)^{+1}$. In this case, note that $p<q$. The decomposing process is as follows:

$$
\begin{gathered}
L(8,11)^{+1} \rightarrow D_{2}^{+1} \rightarrow L(8,5)^{-1} \rightarrow \sigma_{2}^{-2} \sigma_{4}^{-2} D_{0}^{+1} \rightarrow L(2,5)^{+1} \rightarrow D_{2}^{+1} \\
\rightarrow L(2,1)^{+1} \rightarrow D_{-1}^{+1} \rightarrow L(0,1)^{-1} \rightarrow \sigma_{2}^{-1} \sigma_{4}^{-1} \rightarrow L(0,1) .
\end{gathered}
$$


Then we have that

$$
L(8,11)^{+1} \rightarrow \sigma_{2}^{-1} \sigma_{4}^{-1} D_{-1}^{+1} D_{2}^{+1} \sigma_{2}^{-2} \sigma_{4}^{-2} D_{0}^{+1} D_{2}^{+1} \rightarrow L(0,1)
$$

(see Figure 7). Hence, we have the following decomposition of $h$ given by $h\left(w_{i}\right)=$ $K_{i}(i=1,2)$ such that

$$
\begin{aligned}
h= & D_{2}^{-1} D_{0}^{-1} \sigma_{2}^{2} \sigma_{4}^{2} D_{2}^{-1} D_{-1}^{-1} \sigma_{2} \sigma_{4} \\
= & \left(\sigma_{5}^{-2} \sigma_{4}^{-1} \sigma_{5}^{-2} \sigma_{4}^{-2} \sigma_{5}^{-2} \sigma_{4}^{-1} \sigma_{5}^{-2}\right)\left(\sigma_{1}^{-1} \sigma_{2}^{-1} \sigma_{3}^{-1} \sigma_{5} \sigma_{4}^{-1} \sigma_{5}^{-1} \sigma_{3} \sigma_{2} \sigma_{1}\right) \sigma_{2}^{2} \sigma_{4}^{2} \\
& \left(\sigma_{5}^{-2} \sigma_{4}^{-1} \sigma_{5}^{-2} \sigma_{4}^{-2} \sigma_{5}^{-2} \sigma_{4}^{-1} \sigma_{5}^{-2}\right)\left(\sigma_{5}^{-1} \sigma_{4}^{-1} \sigma_{3} \sigma_{2} \sigma_{1}^{-1} \sigma_{2}^{-1} \sigma_{3}^{-1} \sigma_{4} \sigma_{5}\right) \sigma_{2} \sigma_{4} .
\end{aligned}
$$
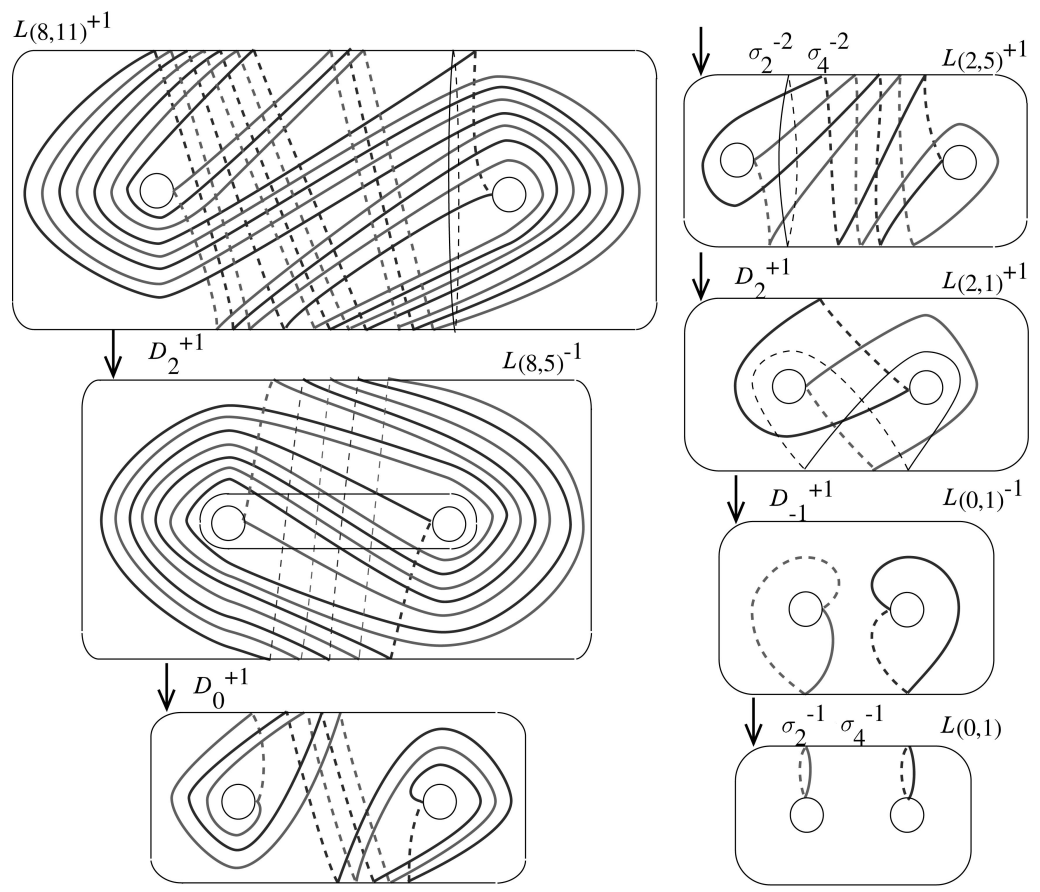

FiguRE $7 . L(8,11)^{+1}$.

We made a computer program to decompose such homeomorphisms above mentioned into canonical Dehn twists and the program can be obtained by anonymous ftp from ics.nara-wu.ac.jp, in directory /export2/ftp/pub/ochiai. Furthermore, we are now developing computer software to analyze the case of higher genus and the results will be published in a forthcoming paper.

\section{REFERENCES}

[L1] W. B. R. Lickorish, A Representation of Orientable Combinatorial 3-Manifolds, Annals of Math. 76 (1962), 531-540. MR 27:1929]

[L2] W. B. R. Lickorish, A finite set of generators for the homeotopy group of a 2-manifold, Proc. Camb. Phil. Soc. 60 (1964), 769-778. MR 30:1500 
[L3] W. B. R. Lickorish, On the Homeotopy Group of a 2-Manifold (corrigendum), Proc. Camb. Phil. Soc. 62 (1966), 679-681. MR 34:799

[O] M. Ochiai, Heegaard Diagrams of 3-Manifolds, Trans. Amer. Math. Soc. 328 (1991), 863-879. MR 92c:57016

Department of Information and Computer Sciences, Faculty of Science, Nara Women's University, NARA 630, JAPAN

E-mail address: ysakata@isc.chubu.ac.jp

Department of Information and Computer Sciences, Faculty of Science, Nara Women's University, NARA 630, JAPAN

E-mail address: ochiai@ics.nara-wu.ac.jp

Department of Information and Computer Sciences, Faculty of Science, Nara WOMEN's University, NARA 630, JAPAN 